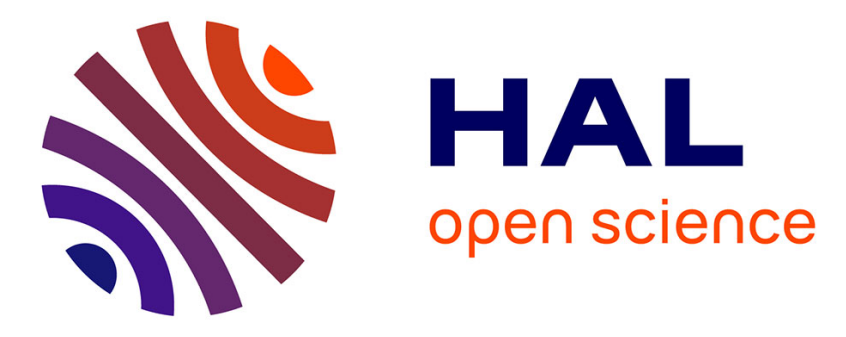

\title{
Bremsstrahlung X-rays as a non-invasive tool for ion beam monitoring
}

Flavien Ralite, Charbel Koumeir, Arnaud Guertin, Ferid Haddad, Quentin

Mouchard, Noël Servagent, Vincent Métivier

\section{To cite this version:}

Flavien Ralite, Charbel Koumeir, Arnaud Guertin, Ferid Haddad, Quentin Mouchard, et al.. Bremsstrahlung X-rays as a non-invasive tool for ion beam monitoring. Nuclear Instruments and Methods in Physics Research Section B: Beam Interactions with Materials and Atoms, 2021, 500-501, pp.76-82. 10.1016/j.nimb.2021.05.013 . hal-03260027

\section{HAL Id: hal-03260027 https://hal.science/hal-03260027}

Submitted on 15 Feb 2022

HAL is a multi-disciplinary open access archive for the deposit and dissemination of scientific research documents, whether they are published or not. The documents may come from teaching and research institutions in France or abroad, or from public or private research centers.
L'archive ouverte pluridisciplinaire HAL, est destinée au dépôt et à la diffusion de documents scientifiques de niveau recherche, publiés ou non, émanant des établissements d'enseignement et de recherche français ou étrangers, des laboratoires publics ou privés. 


\title{
BREMSSTRAHLUNG X-RAYS AS A NON-INVASIVE TOOL FOR ION BEAM MONITORING
}

\author{
Flavien Ralite ${ }^{(1,3, *)}$, Charbel Koumeir ${ }^{(2)}$, Arnaud Guertin ${ }^{(1)}$, Férid Haddad ${ }^{(1,2)}$, Vincent Métivier ${ }^{(1)}$, Quentin \\ Mouchard $^{(1)}$, Noël Servagent ${ }^{(1)}$ \\ ${ }^{(1)}$ Laboratoire SUBATECH, IMT Atlantique, CNRS/IN2P3, Université de Nantes, Nantes, France \\ ${ }^{(2)}$ GIP ARRONAX, Saint-Herblain, France \\ ${ }^{(3)}$ Institut Bergonié, Department of radiotherapy, Bordeaux, France \\ *Corresponding author: flavien.ralite@ subatech.in2p3.fr
}

\begin{abstract}
Non-invasive beam monitoring tools are required to guarantee the delivered dose during ion irradiation. Measuring bremsstrahlung X-rays emitted directly from the irradiated medium is a promising approach. This work presents the feasibility of the method for different incident particles with different energies. A PMMA target was bombarded with helium ions of $68 \mathrm{MeV}$ and protons beams of 30, 40, and $50 \mathrm{MeV}$. The bremsstrahlung signal, measured with a SDD, was in the magnitude of $10^{4} \mathrm{X}$-rays $/ \mathrm{nC}$, indicating the significant sensitivity of the method. A good agreement was found $(<6 \%)$ between the experimental and simulation bremsstrahlung spectra. For a thick target, the bremsstrahlung yield saturates and is directly proportional to the beam energy and the deposited dose at the surface. In addition, the bremsstrahlung spectrum shape contains information about the beam energy. Therefore, the bremsstrahlung signal gives access to the deposited dose and beam energy with precision depending on the experimental uncertainties.
\end{abstract}

Keywords : X-rays, Bremssstrahlung, Ion beam monitoring, Radiobiology, Dosimetry

\section{INTRODUCTION}

Ion beam therapy is currently in development, especially proton, helium, and carbon beams [1-6]. To improve the treatment quality, the determination of the biological dose induced by ion beams is a challenge [7,8]. Then, radiobiology experiments on cells are necessary to compare with the predictions of the biological models [9-11].

In this frame, the ARRONAX cyclotron develops a platform for radiobiology experiments on cells with proton and helium beams with energies up to $70 \mathrm{MeV}$ [12,13]. Ion beam monitoring tools are mandatory to guarantee the dose delivered to biological samples. The ionization chambers and X-ray fluorescence from thin metallic foils can be used to monitor the beam [12]. When crossing the monitor material thicknesses, the beam undergoes a dispersion that leads to a lateral spreading at the sample position particularly in the case of low energy beams. A Faraday cup [14] is adapted to monitor the beam intensity when the beam crosses a thin sample. Non-invasive methods exist like the Ar fluorescence from the air to monitor the beam intensity of the incident beam [15]. It requires less room in the beam path and avoids material activation and damage induced by the irradiation. Nevertheless, this method does not give information from the irradiated medium. Noninvasive methods using radiations emitted from the irradiated medium were developed in the context of hadrontherapy to monitor the beam range, such as prompt gamma measurements [16-18], PET acquisitions [19], and bremsstrahlung radiation measurements [20-23]. This work presents a noninvasive beam monitoring method based on the detection of the bremsstrahlung X-rays, emitted by the irradiated medium, to monitor the ion beam for the radiobiology experiments.

Previous works performed at the ARRONAX facility proved the capability of bremsstrahlung X-rays to monitor the deposited 
dose induced by an alpha beam of $68 \mathrm{MeV}$ [24]. The sensitivity found with an HPGe detector was around $90 \mathrm{X}$-rays/Gy. The present work follows the ones of Schwob et al [24]. We modified the experimental set-up to increase the number of detected photons emitted from a Polymethyl Methacrylate (PMMA) target used as a biological medium surrogate thanks to its effective atomic number close to water. Additional particle beams like protons with different energies were experimentally investigated and compared with simulations derived from theoretical works [25-29]. The dependence of the bremsstrahlung signal with the medium thickness and the beam energy was studied. The latter properties are of importance to monitor the deposited dose and the beam energy into radiobiology experiments [24]. The beam energy dependence of the bremsstrahlung spectrum shape was also studied.

\section{MATERIALS AND METHODS}

\section{Simulation of the Bremsstrahlung spectrum}

A homemade program using python (v.3.7), simulates the bremsstrahlung spectrum. The next sub-sections describe step by step how the model was built.

The simulation of the bremsstrahlung spectrum is based on equation (1) as follows:

$$
\begin{aligned}
& N_{X}^{B r}=N_{p} \cdot \iint_{E_{p}^{i} h v}^{E_{p}^{t}} \varepsilon(h v) \cdot \frac{d^{2} \sigma^{B r}}{d \Omega d h v}\left(E_{p}, h v, \theta\right) . \\
& \mathcal{A}(d, h v, \theta) d h v d E_{p},
\end{aligned}
$$

where $N_{X}^{B r}$ is the number of bremsstrahlung $\mathrm{X}$-rays for a given photon energy $h v, N_{p}$ is the number of incident ions, $E_{p}{ }^{i}$ and $E_{p}{ }^{t}$ are respectively the initial and the transmitted energies of the projectile through the PMMA target. $\varepsilon(h v)$ is the detection efficiency of the silicon drift detector (SDD), $\quad \sigma^{B r}(E, h v, \theta)$ corresponds to the bremsstrahlung cross section and $\mathcal{A}(d, h v, \theta)$ is the $\mathrm{X}$-rays attenuation through the medium.

\section{Bremsstrahlung cross section}

The total cross section of the bremsstrahlung $\sigma^{B r}$ is the sum of three different processes : QFEB (Quasi Free Electron Bremsstrahlung), SEB (Secondary Electron Bremsstrahlung) and AB+RI (Atomic Bremsstrahlung and Radiative Ionisation) [25-29]. $\frac{d^{2} \sigma^{B r}}{d \Omega d h v}\left(E_{p}, h v, \theta\right)=\frac{d^{2} \sigma^{Q F E B}}{d \Omega d h v}+\frac{d^{2} \sigma^{S E B}}{d \Omega d h v}+\frac{d^{2} \sigma^{A B+R I}}{d \Omega d h v}$.

The cross section of every bremsstrahlung component, i, can be expressed as the product of two factors called strength $F_{1}$ and shape $F_{2}$ factors. The strength factor depends on $E_{p}, Z_{p}$ (energy and atomic number of the projectile), and $Z_{T}$ (atomic number of the target) whereas the shape factor depends on the angle of X-ray emission $\theta$.

$\frac{d^{2} \sigma^{i}}{d \Omega d h v}=F_{1}\left(E_{p}, h v, Z_{p}, Z_{T}\right) \cdot F_{2}(\theta)$.

Theoretical cross-sections of bremsstrahlung are given by the work of Ishii et al [25,29].

\section{Energy losses of ions in the PMMA target}

The stopping powers for helium ions and protons are extracted respectively from the ASTAR and PSTAR databases [29]. The PMMA target was numerically divided into $100 \mu \mathrm{m}$ thick slices, for which the variation of the cross-section with the beam energy is considered negligible.

\section{Medium attenuation}

The X-ray mass attenuation database [30] was used to calculate the X-ray attenuation in the PMMA target. The coefficient attenuation is calculated by equation (4). $t_{\text {PMMA }}$ and $\mathrm{d}_{\text {PMMA }}$ are, respectively, the PMMA slice thickness and its depth inside the target.

$$
\begin{aligned}
& \mathcal{A}(d, h v, \theta)= \\
& \exp \left(\frac{-\mu_{P M M A} \cdot d_{P M M A}}{\cos (\theta)}\right) \int_{0}^{t_{P M M A}} \exp \left(\frac{-\mu_{P M M A} \cdot x}{\cos (\theta)}\right) d x .
\end{aligned}
$$

\section{Detection efficiency}

The differential bremsstrahlung cross section $\frac{d^{2} \sigma^{B r}}{d \Omega d h v}$ decreases while the photon energy $h v$ increases $[25,29]$.To improve the statistics of the detected X-rays, the detection of low-energy photons has to be maximized. The SDD seems well adapted as its detection efficiency is optimal between 3 and $14 \mathrm{keV}$ (as shown in Figure 1) [24]. The SDD detector efficiency is determined according to the model of Mohanty et al [31]. The model inputs are the X-ray mass attenuation coefficients [30] and the detector specificities. The latter are the active detector area, $30 \mathrm{~mm}^{2}$, the detector thickness, $450 \mu \mathrm{m}$, and the beryllium entrance window, $16 \mu \mathrm{m}$. The Nelson Blachman formula [31] gives the fractional solid angle in our simulation. 


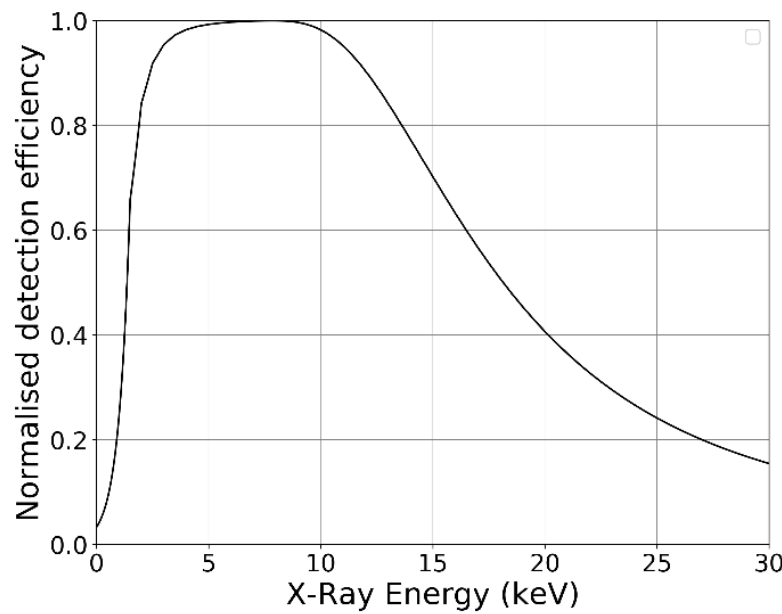

Fig. 1. The normalized detection efficiency of our silicon drift detector (SDD) without taking into account the air attenuation between the target and the detector.

\section{Experimental set-up}

The ARRONAX cyclotron (IBA C70) [13] delivered an alpha beam of $68 \mathrm{MeV}$ and proton beams of 30, 40, and $50 \mathrm{MeV}$ in air through an exit window made of a $50 \mu \mathrm{m}$ thick Kapton foil. Some PMMA targets with thicknesses of $500 \mu \mathrm{m}$, $1000 \mu \mathrm{m}, 1500 \mu \mathrm{m}$, and $3000 \mu \mathrm{m}$ were irradiated.

As shown in Figure 2, an Al collimator ( $5 \mathrm{~mm}$ diameter) narrowed the beam on the target. The beam exit window-target distance was $23 \mathrm{~cm}$. The target-detector distance was $5 \mathrm{~cm}$, which allowed the detection of low energy photons (but $>3 \mathrm{keV}$ ) emitted by the medium. The angle between the detector axis and the incident beam is $135^{\circ}$. The efficiency model presented above was checked with calibrated standard sources $\left({ }^{241} \mathrm{Am}\right.$, ${ }^{109} \mathrm{Cd}$ and ${ }^{55} \mathrm{Fe}$ ). The deviation between the model and the experimental efficiencies is below $3 \%$.

For each particle and each energy, the background spectrum was measured before the irradiation and subtracted from the measured PMMA spectra to extract the bremsstrahlung signal.

\section{Beam intensity monitoring}

A Faraday cup, with a thin Kapton window $(25 \mu \mathrm{m}$ thick) and located behind the target, can measure the beam charge with a relative error of less than $1 \%$ in the beam intensity range of about $100 \mathrm{pA}$ (intensity used during the experiments presented in this work). Due to the beam/target interaction, a thick target increases the angular straggling of the beam [32,33]. Thus, the beam size will be larger than the Faraday cup window, and only a part of the beam charge is collected. In this case, the Faraday cup is no longer useful for measuring the beam fluence during irradiation of the target. Therefore, a beam monitor before the beam passing through the target is necessary. In our case, the characteristic X-rays of argon emission $(2.9 \mathrm{keV})$, resulting from the interaction of the incident charged particles with the argon atoms present in the air in front of the target, can be detected with the silicon detector. To calibrate the argon X-rays signal, the entrance window of the Faraday cup was placed precisely at the same position as the PMMA target. An acquisition without the target is realized to measure both the beam fluence with the Faraday cup and the number of X-rays from the argon emission with the SDD

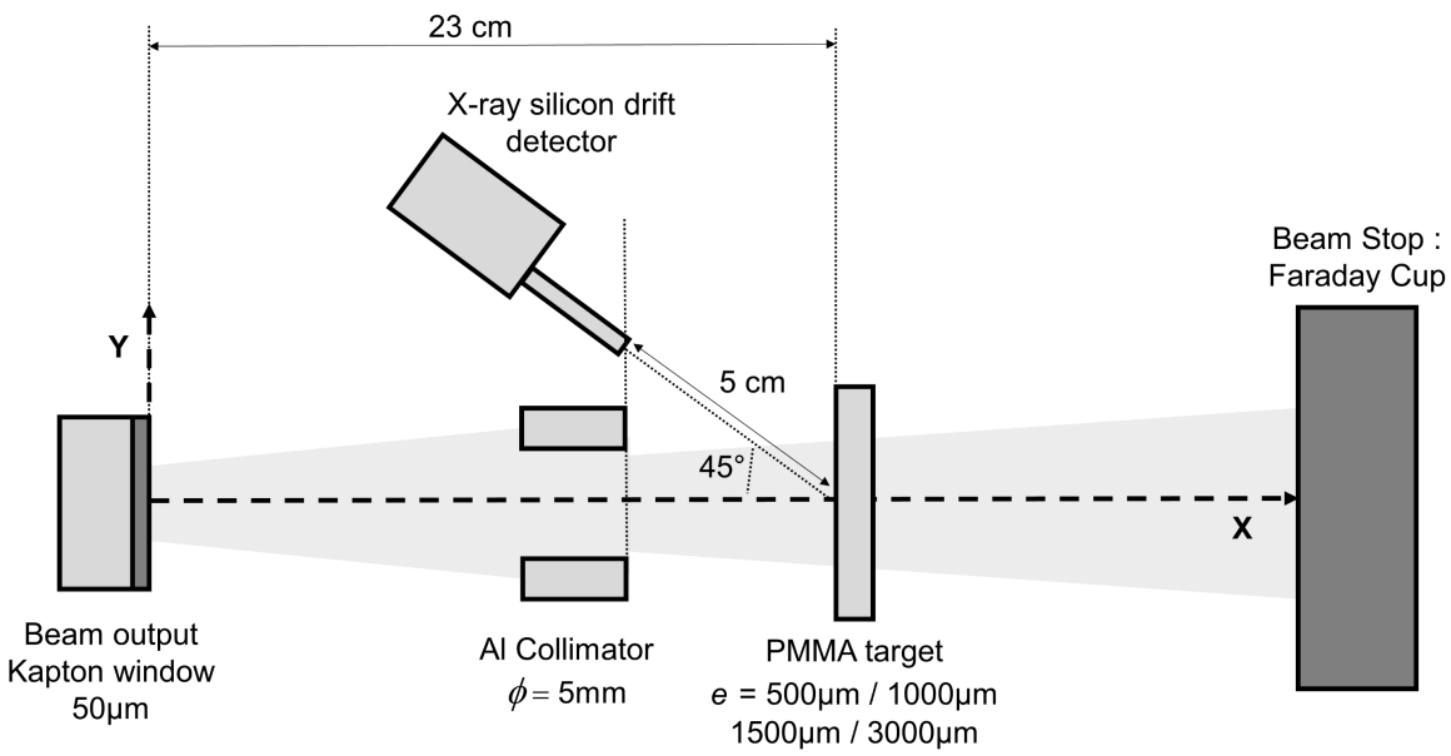

Fig. 2. Scheme of the experimental set-up. 
detector. The number of incident charged particles $\mathrm{Np}$ is related to the collected charge Q by :

$N_{p}=\frac{Q}{Z_{p} \cdot e}$.

The integration of the argon peak $(2.9 \mathrm{keV})$ in the spectrum gives the number of $\mathrm{X}$-rays from the argon. The ratio between the beam fluence and the number of X-rays from argon determines a calibration factor. The uncertainty, which includes statistical and experimental uncertainties, was about $5 \%$. This calibration factor gives the number of incident particles from the number of X-rays from argon registered during the PMMA target irradiations.

\section{Dose determination}

The delivered dose $D$ to the surface layer of the medium is given by the equation (6).

$D[G y]=\phi\left[\mathrm{cm}^{-2}\right] \cdot \operatorname{LET}\left[\frac{\mathrm{MeV} . \mathrm{cm}^{2}}{\mathrm{~g}}\right] \cdot 1.6 * 10^{-10}$,

where $\phi$ is the fluence of charged particles, which is the ratio of the number of charged particles $N_{p}$ with the beam spot area. The LET is the linear energy transfer of the incident ions at the surface target determined thanks to the PSTAR/ASTAR tables [34]. The factor $1.6 \cdot 10^{-10}$ converts the equation into SI-units $(\mathrm{MeV} / \mathrm{g}$ to $\mathrm{J} / \mathrm{kg}$ ).

\section{RESULTS}

Bremsstrahlung spectrum from the PMMA target

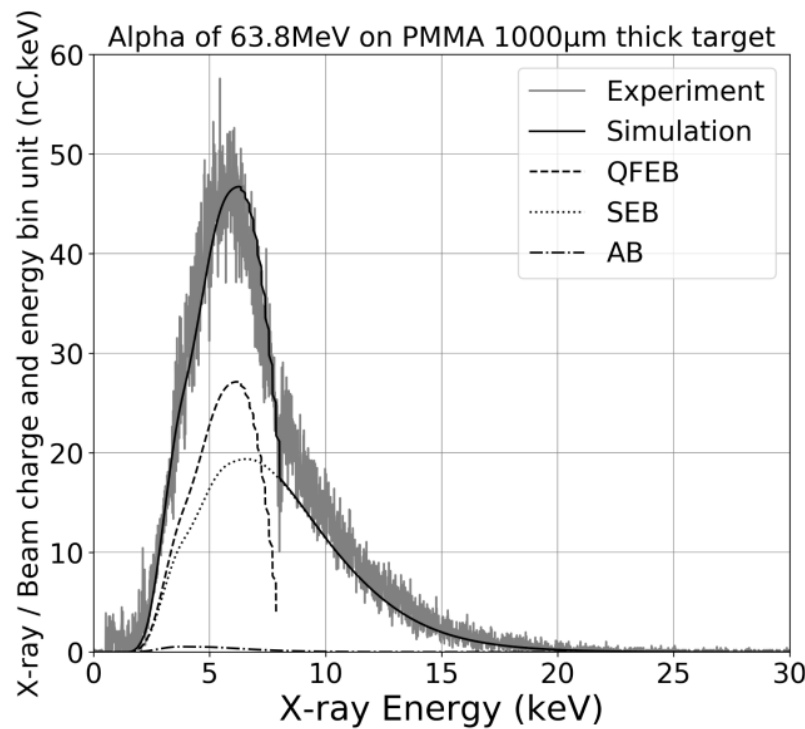

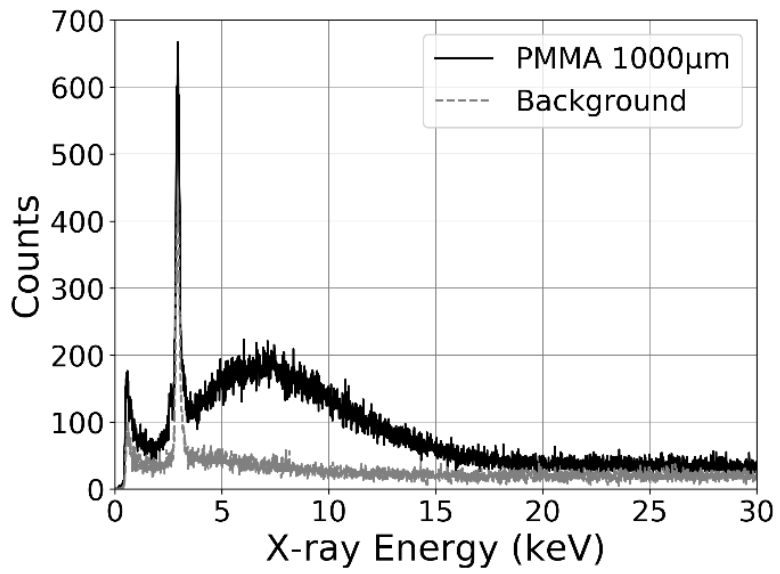

Fig. 3. Raw spectra measured with $40 \mathrm{MeV}$ protons for a $1000 \mu \mathrm{m}$ thick PMMA target (black) and without target (background, grey). The measured beam fluences are given in table 1 .

Figure 3 presents the $\mathrm{X}$-rays raw spectra obtained for the PMMA target (1000 $\mu \mathrm{m}$ thick) and the background (no target) for a $40 \mathrm{MeV}$ proton beam. Both include the characteristic peak of argon $(2.9 \mathrm{keV})$. An escape peak is present at the energy of $1.16 \mathrm{keV}$ corresponding to the energy difference between $2.9 \mathrm{keV}$ (K-X-ray of Ar) and $1.74 \mathrm{keV}(\mathrm{K}$ $\mathrm{X}$-ray of $\mathrm{Si}$ ). The difference in the continuous component of both spectra shows clearly the bremsstrahlung radiations coming from the PMMA target.

\section{Comparison of the simulated and the experimental spectra}

Figure 4 presents the simulated and measured bremsstrahlung spectra for a $1000 \mu \mathrm{m}$

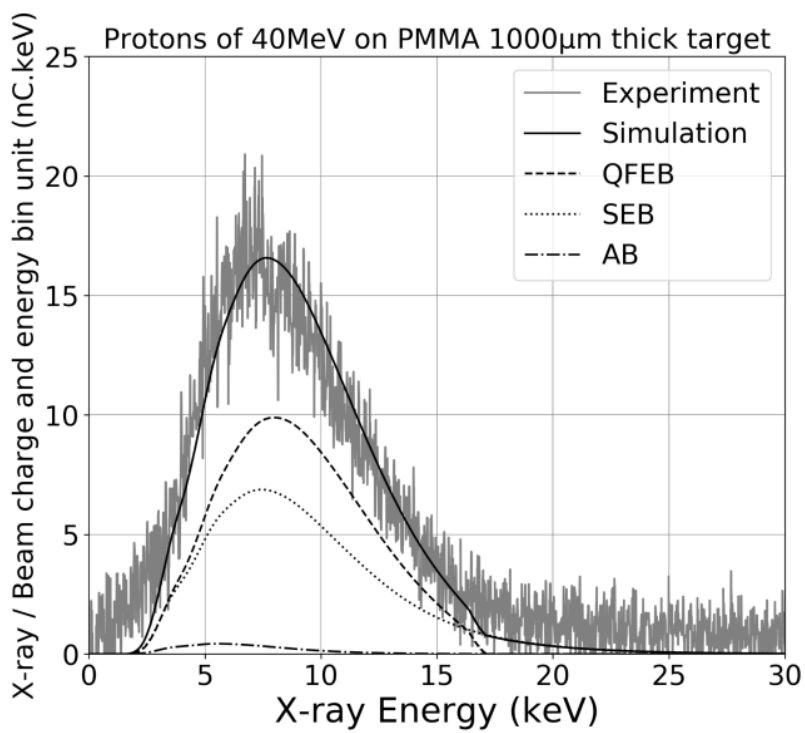

Fig. 4. Measured and simulated bremsstrahlung spectra for $63.8 \mathrm{MeV}$ alpha particles (left) and $40 \mathrm{MeV}$ protons (right) interacting with a $1000 \mu \mathrm{m}$ PMMA thick target. The measured beam fluences are given in table 1. 


\begin{tabular}{|c|c|c|c|c|c|c|c|c|}
\hline Particle & $\begin{array}{c}\text { Beam } \\
\text { energy } \\
\text { at the } \\
\text { target } \\
\text { entrance } \\
(\mathrm{MeV})\end{array}$ & $\begin{array}{l}\text { Beam } \\
\text { energy } \\
\text { at the } \\
\text { target } \\
\text { exit } \\
(\mathrm{MeV})\end{array}$ & $\begin{array}{l}\text { Target- } \\
\text { detector } \\
\text { distance } \\
(\mathrm{cm})\end{array}$ & $\begin{array}{l}\text { Beam fluence } \\
\text { measured } \\
\text { (nC bkg) } \\
\text { (nC target) }\end{array}$ & $\begin{array}{c}\text { Dose } \\
\text { conversion } \\
\text { factor* } \\
(\mathrm{Gy} / \mathrm{nC})\end{array}$ & $\begin{array}{c}\text { Bremsstrahlung } \\
\text { yield, measured } \\
(\mathrm{X}-\mathrm{rays} / \mathrm{nC})\end{array}$ & $\begin{array}{c}\text { Bremsstrahlung } \\
\text { yield, simulated } \\
(\mathrm{X} \text {-rays/nC) }\end{array}$ & $\begin{array}{l}\text { Relative } \\
\text { difference } \\
\quad(\%)\end{array}$ \\
\hline Alpha & 63.8 & 47.9 & 5 & $\begin{array}{l}10.22 \pm 0.102 \\
5.036 \pm 0.252\end{array}$ & 215 & $25270 \pm 1482$ & 24070 & $\begin{array}{l}4.98 \\
5.40\end{array}$ \\
\hline Alpha & 63.8 & 47.9 & 2.7 & $\begin{array}{l}3.497 \pm 0.035 \\
3.562 \pm 0.178\end{array}$ & 215 & $221695 \pm 12935$ & 209625 & $\begin{array}{l}5.76 \\
5.74\end{array}$ \\
\hline Proton & 30.1 & 27.7 & 5 & $\begin{array}{l}12.09 \pm 0.121 \\
3.497 \pm 0.175\end{array}$ & 65 & $12195 \pm 719$ & 12743 & $\begin{array}{l}4.49 \\
4.23\end{array}$ \\
\hline Proton & 39.3 & 37.5 & 5 & $\begin{array}{l}5.128 \pm 0.052 \\
7.786 \pm 0.389\end{array}$ & 52 & $14204 \pm 829$ & 13435 & $\begin{array}{l}5.72 \\
5.00\end{array}$ \\
\hline Proton & 49.6 & 48.1 & 5 & $\begin{array}{l}7.489 \pm 0.075 \\
4.850 \pm 0.242\end{array}$ & 43 & $13656 \pm 800$ & 13150 & $\begin{array}{l}3.84 \\
3.60\end{array}$ \\
\hline
\end{tabular}

Table 1. Measured and simulated bremsstrahlung yields for alpha particles and protons for a PMMA target with a thickness of $1000 \mu \mathrm{m}$. *The equation (6) using the ASTAR/PSTAR database gives the dose conversion factor for 1 $\mathrm{nC}$ and a beam spot area of $5 \mathrm{~mm}^{2}$.

thick PMMA target irradiated with $63.8 \mathrm{MeV}$ alpha particles (left) and $40 \mathrm{MeV}$ protons (right). The figure also includes different bremsstrahlung components (SEB, QFEB and AB).

The simulated data fit the experimental spectra for the alpha and the proton beams quite well. The bremsstrahlung signal comes mainly from QFEB and SEB. The inflection point in the simulated spectra corresponds to the transition of the predominance from QFEB to SEB. As described in the bremsstrahlung cross section models [25], the maximum energy of the QFEB is proportional to the ratio between the beam energy and the projectile mass, explaining the difference of the energy transition in the alpha and proton spectra.
These results indicate the validity of the bremsstrahlung cross section models for helium ions and protons in the range of the beam energies used in this work.

\section{The sensitivity of the bremsstrahlung signal}

The integral of the number of X-rays, between 1 and $30 \mathrm{keV}$ in each spectrum, is used to evaluate the bremsstrahlung yield (bremsstrahlung intensity for a given number of incident beam particles). Results are given in Table 1, dealing with the number of bremsstrahlung X-rays detected (per beam charge unit) for a PMMA target of $1000 \mu \mathrm{m}$ thick. The uncertainties on the measured bremsstrahlung yield (around 6\%) mainly come
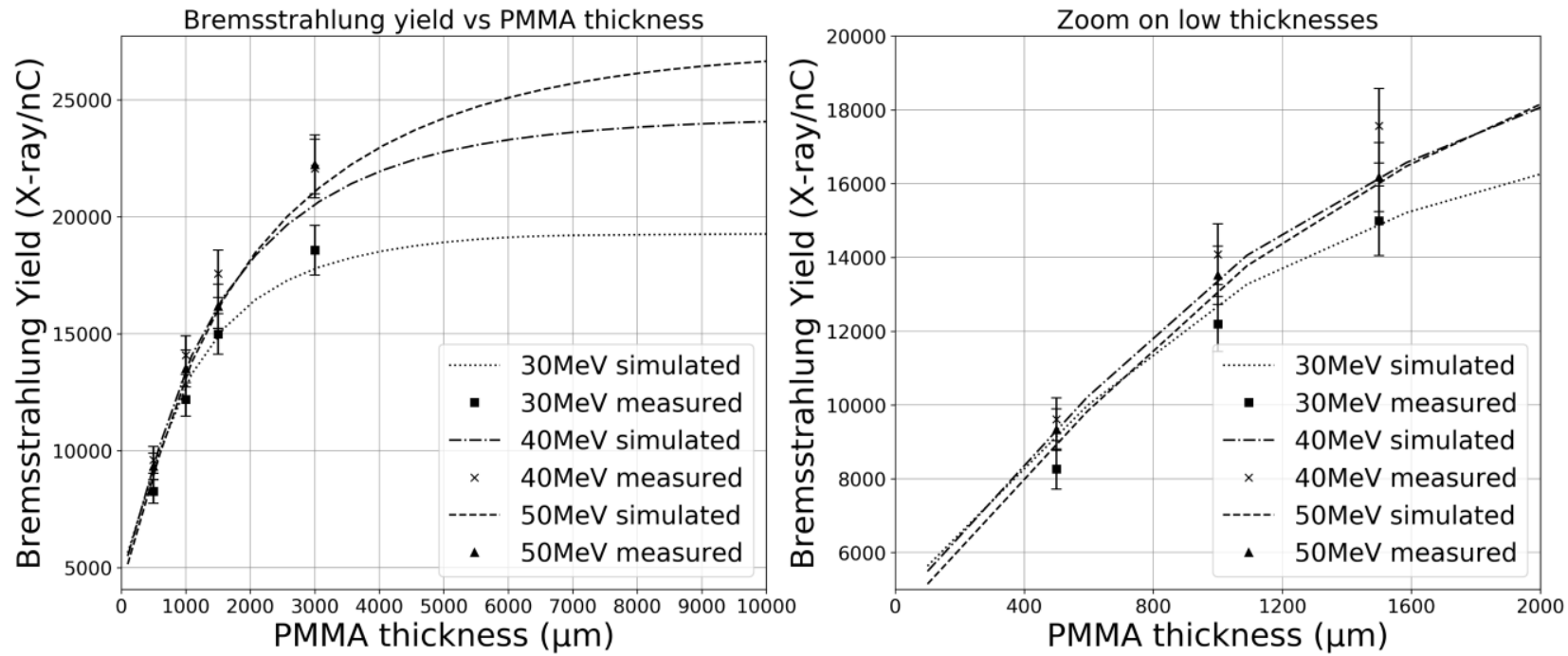

Fig. 5. The bremsstrahlung yield versus the PMMA thickness with proton beams. Solid and dashed lines represent the simulations whereas markers correspond to experimental data. The right figure corresponds to a Zoom of the left figure at low thicknesses. The measured beam fluences were between $(3.511 \pm 0.203) \mathrm{nC}$ and $(6.780 \pm 0.393) \mathrm{nC}$. 

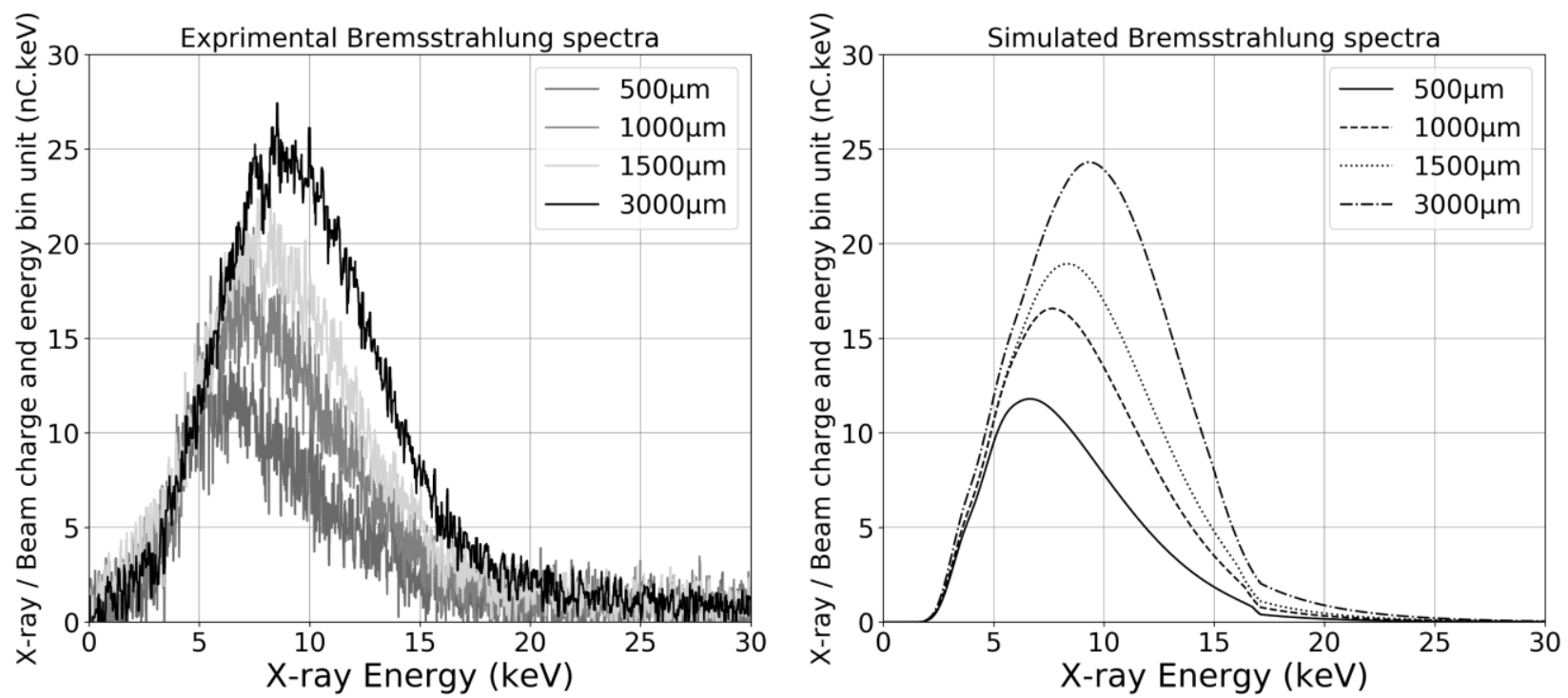

Fig. 6. The measured (left) and simulated (right) bremsstrahlung spectra for $40 \mathrm{MeV}$ protons on PMMA targets with different thicknesses $(500,1000,1500$, and $3000 \mu \mathrm{m})$. The measured beam fluences were between $(3.511 \pm 0.203) \mathrm{nC}$ and $(6.780 \pm 0.393) \mathrm{nC}$.

from the measure of the beam fluence. The statistical errors can be neglected.

The measured bremsstrahlung yield is in the magnitude of $2.10^{4} \mathrm{X}$-rays $/ \mathrm{nC}$ and $1.10^{4} \mathrm{X}$ rays/nC, respectively, for alpha and proton beams. The bremsstrahlung cross-sections of QFEB and SEB are higher for the alpha beam explaining the difference with the proton bremsstrahlung yield.

The relative difference between measurements and simulations ranges from $3 \%$ to $6 \%$, in the same magnitude than the error bars, and shows a good behavior of the bremsstrahlung model. The differences come from the uncertainties of the experimental setup (detection efficiency and beam fluence), and the nuclear background induced by the PMMA target (not included in the simulation model). Ishii et al. [29] also observed this difference between the model and the experimental spectra for photons with an energy greater than $15 \mathrm{keV}$ because of the Compton tail.

An acquisition for the alpha particles, with a distance of $2.7 \mathrm{~cm}$ between the target and the detector, was performed. The bremsstrahlung yield increases by a factor of 10 due to the shorter distance that increases the solid angle and reduces the air attenuation of low-energy photons. Our improved experimental setup (using the SDD detector and reducing the target-detector distance to $2.7 \mathrm{~cm}$ ) increases the bremsstrahlung yield by promoting the low-energy $\mathrm{X}$-ray detection. It also points out that the bremsstrahlung yield is high enough to monitor the delivered dose in radiobiology experiments (about $1000 \mathrm{X}$-rays/Gy).

\section{The bremsstrahlung yield saturation}

Figure 5 shows the variations in the bremsstrahlung yield as a function of the PMMA target thickness for different proton beam energies. Solid and dotted lines represent simulation results, while markers correspond to experimental data. According to the simulations, the bremsstrahlung yield saturates for a fixed target thickness for a given beam. In thinner targets, the bremsstrahlung yield increases with the thickness that produces more bremsstrahlung radiations. The mediumdepth is not too large to attenuate all the created Xrays, and the bremsstrahlung radiations can escape through the PMMA target and reach the detector.

Figure 6 shows this phenomenon using measured data (left) and simulated spectra (right) in the case of the interaction of a $40 \mathrm{MeV}$ proton beam on PMMA targets of different thicknesses $(500 \mu \mathrm{m}$, $1000 \mu \mathrm{m}, 1500 \mu \mathrm{m}$, and $3000 \mu \mathrm{m})$. The number of detected X-rays grows with the target thickness, but not linearly. The difference between the spectra corresponding to $500 \mu \mathrm{m}$ and $1000 \mu \mathrm{m}$ is larger than the difference between $1000 \mu \mathrm{m}$ and $1500 \mu \mathrm{m}$ due to the saturation effect.

The produced bremsstrahlung X-rays from a given PMMA slice, located in-depth, shifts to lower energies because of the beam slowing down.

Therefore, two combined phenomena, the medium attenuation and the shifting of the bremsstrahlung spectrum, explain the decrease and the saturation of the bremsstrahlung yield. In the bremsstrahlung yield saturation case, the number of detected photons is proportional to the number of incident ions, and by extension, to the deposited 


\begin{tabular}{ccccccccc}
\hline $\begin{array}{c}\text { Beam } \\
\text { energy } \\
\text { estimated } \\
\text { at the } \\
\text { target } \\
\text { surface } \\
(\mathrm{MeV})\end{array}$ & $\begin{array}{c}\text { Theoretical } \\
\text { beam } \\
\text { energy at } \\
\text { the target } \\
\text { surface } \\
(\mathrm{MeV})\end{array}$ & $\begin{array}{c}\text { Relative } \\
\text { difference } \\
\text { on the } \\
\text { beam } \\
\text { energy } \\
(\%)\end{array}$ & $\begin{array}{c}\text { X-ray } \\
\text { mean } \\
\text { energy } \\
\begin{array}{c}E_{\text {mean }} \\
\text { estimated } \\
(\mathrm{keV})\end{array}\end{array}$ & $\begin{array}{c}\text { X-ray } \\
\text { mean } \\
\text { energy } \\
\begin{array}{c}\mathrm{E}_{\text {mean }} \\
\text { calculated } \\
(\mathrm{keV})\end{array}\end{array}$ & $\begin{array}{c}\text { Relative } \\
\text { difference } \\
\text { on Emean } \\
(\%)\end{array}$ & $\begin{array}{c}\text { FWHM } \\
\text { estimated } \\
(\mathrm{keV})\end{array}$ & $\begin{array}{c}\text { FWHM } \\
\text { calculated } \\
(\mathrm{keV})\end{array}$ & $\begin{array}{c}\text { Relative } \\
\text { difference } \\
\text { on } \\
\text { FWMH } \\
(\%)\end{array}$ \\
\hline $40.5 \pm 0.1$ & 30.1 & 5.61 & 7.360 & 7.431 & 0.96 & 6.944 & 7.147 & 2.92 \\
\hline $51.9 \pm 0.1$ & 39.3 & 3.05 & 7.715 & 7.694 & 0.26 & 7.907 & 7.846 & 0.77 \\
\hline
\end{tabular}

Table 2. Estimation of the ion beam energy at the target surface from the X-ray mean energy $E_{\text {mean }}$ and the FWHM of the X-rays spectra plotted in Figure 7. The calculated data were determined with the simulated spectrum for the theoretical beam energy (PSTAR). The estimated data were determined with the least square minimization between the measured spectra and a database of simulated spectra, made with different beam energies (with a bin of $0.1 \mathrm{MeV}$ ). Relative differences are the difference between the experimental spectra and the simulated spectra with the best fit.

dose according to equation (6). Then, the monitoring of the deposited dose is possible.

\section{Beam energy monitoring}

In bremsstrahlung saturation conditions, the number of detected X-rays depends on the ion beam energy (Figure 5). The higher the incident proton energy, the higher the PMMA thickness required to reach the bremsstrahlung saturation. When the saturation is reached, the simulation tendency (figure 5) shows that it is possible to dissociate two beams with a difference of $10 \mathrm{MeV}$ by taking into account the error bars. However, reducing the experimental errors can improve beam energy monitoring. Uncertainties are mainly from the beam fluence measurement (about 6\%). The latter can be improved by using a second X-rays

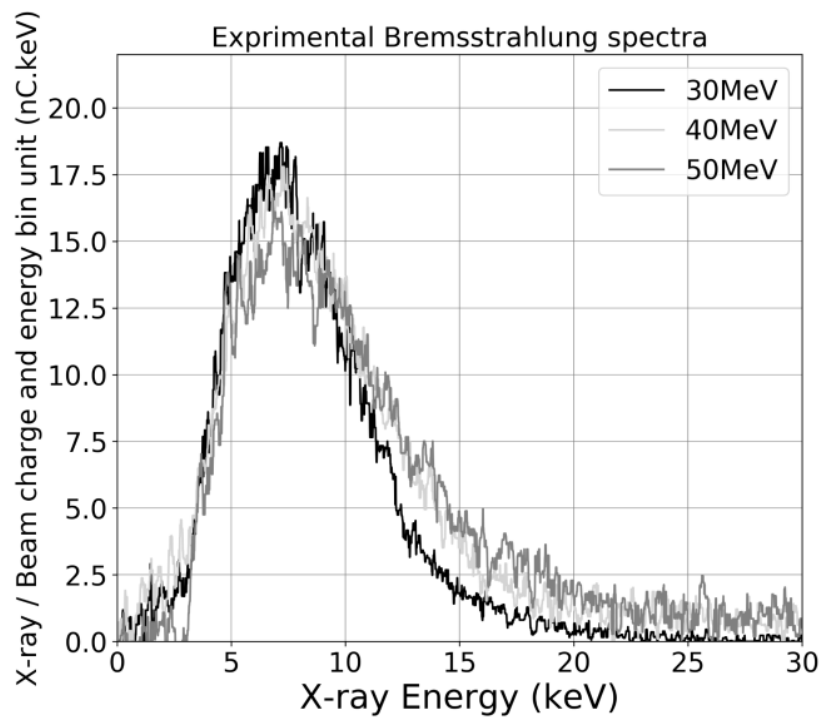

Fig. 7. The measured (left) and simulated (right) bremsstrahlung spectra for protons of different energies $(30 \mathrm{MeV}, 40 \mathrm{MeV}$, and $50 \mathrm{MeV})$ interacting on a $1000 \mu \mathrm{m}$ thick PMMA target. The measured beam fluences are given in table 1 .

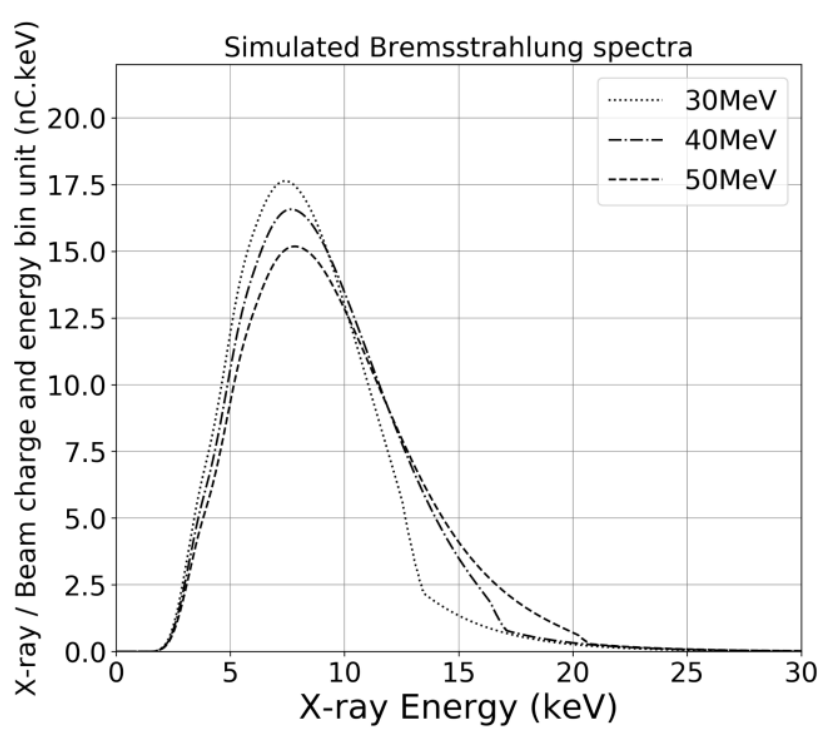

detector dedicated to the detection of argon emissions.

Figure 7 presents the bremsstrahlung spectra measured with proton beams of 30, 40, and $50 \mathrm{MeV}$ for a $1000 \mu \mathrm{m}$ thick PMMA target. These results show the spectrum hardening as a function of the beam energy. The higher the energy of the protons, the lower the number of low-energy photons $(<5 \mathrm{keV})$, conversely to the number of high-energy photons ( $>10 \mathrm{keV})$ detected. The mean energy and the Full Width at Half Maximum (FWMH) extracted from the spectra quantify the spectrum hardening (see Table 2).

The increases of both metrics show the spectrum hardening and differentiate the incident ions energy using the bremsstrahlung spectra analysis. Therefore, the beam energy can be monitored using the characteristics of the 
bremsstrahlung spectra. However, the weak variations of $\mathrm{E}_{\text {mean }}$ and $\mathrm{FWMH}$ limit the dissociation between two beams with close energies. These observations result from the detector efficiency dependence, which does not promote the detection of high-energy photons.

\section{DISCUSSION \& CONCLUSION}

This work investigates the use of the bremsstrahlung spectrum as a non-invasive tool to monitor ion beams in radiobiology experiments. The biological medium surrogate was PMMA.

The whole results presented in this study depend on the experiment conditions: the detector position (viewing the target surface), the targetdetector distance, the PMMA thickness, and the detection efficiency.

The SDD promotes the detection of the Xrays with low-energies and can minimize the targetdetector distance (due to its compact design). These points improve the sensitivity of the measured bremsstrahlung signal, as predicted by Schwob et al [24]. The significant measured signal $\left(>10^{4} \mathrm{X}\right.$ rays/nC) allows the use of the bremsstrahlung $\mathrm{X}$ rays as a non-invasive tool for ion beam monitoring in case of radiobiology experiment. The method presented is not convenient for beam spot scanning due to the compact set-up required. In fact the target-detector distance has to be minimized to improve the statistics of the detected X-rays.

The agreement between the simulations and the measurements validates the model for different beam particles (protons and $\mathrm{He}$ ions) and different energies $\left(\mathrm{He}^{2+} 68 \mathrm{MeV}, \mathrm{H}^{+} 30,40\right.$, and $\left.50 \mathrm{MeV}\right)$.

A bremsstrahlung yield saturation is due to the medium attenuation and the shifting of the bremsstrahlung photons production to lower energies in-depth in the target. Using the silicon drift detector, with a maximum efficiency for low energy photons $<10 \mathrm{keV}$, saturation conditions are obtained with a several $\mathrm{mm}$ target thickness.

The maximum bremsstrahlung yield can monitor the beam intensity and the deposited dose (knowing the LET of the incident charged particles in the medium). The signal saturation can also monitor the beam energy. However, the variation of the spectrum as a function of the beam energy is too weak to dissociate close beam energies $(<10 \mathrm{MeV})$, with the current uncertainties $(6 \%)$. In addition, the bremsstrahlung spectrum characteristics, FWHM and $E_{\text {mean }}$, are related to the beam energy. The bremsstrahlung spectra analysis presented in this work shows that the estimated beam energies are close to those expected with a difference of several
MeV. The energy beam monitoring could be interesting within the radiobiological experiments with a Spread Out Bragg Peak (SOBP) to check the different pristine Bragg peaks [6]. For that, the precision of the energy monitoring has to be increased by improving the experimental set up.

\section{ACKNOWLEDGMENTS}

The authors thank the accelerator group and risk management group of the ARRONAX Public Interest Group (GIP). This project is partially supported by grants from the French National Agency for Research called "Investissements d'Avenir", Equipex Arronax-Plus (ANR-11EQPX-0004), Labex IRON (ANR-11-LABX-1801), and ISITE NExT (ANR-16-IDEX-0007).

\section{REFERENCES}

[1] N. Kanematsu, T. Inaniwa, S. Yonai, H. Mizuno, Technical Note: Reconstruction of physical and biological dose distributions of carbon-ion beam through deconvolution of longitudinal dosimeter responses, Med. Phys. 46 (2019) 1478-1482. https://doi.org/10.1002/mp.13360.

[2] T. Furukawa, Y. Hara, K. Mizushima, N. Saotome, R. Tansho, Y. Saraya, T. Inaniwa, S. Mori, Y. Iwata, T. Shirai, K. Noda, Development of NIRS pencil beam scanning system for carbon ion radiotherapy, Nuclear Instruments and Methods in Physics Research Section B: Beam Interactions with Materials and Atoms. 406 (2017)

$361-367$ https://doi.org/10.1016/j.nimb.2016.10.029.

[3] T. Tessonnier, A. Mairani, W. Chen, P. Sala, F. Cerutti, A. Ferrari, T. Haberer, J. Debus, K. Parodi, Proton and helium ion radiotherapy for meningioma tumors: a Monte Carlo-based treatment planning comparison, $\begin{array}{lllll}\text { Radiat } & \text { Oncol. } & 13 & \text { (2018) }\end{array}$ https://doi.org/10.1186/s13014-017-0944-3.

[4] M. Krämer, E. Scifoni, C. Schuy, M. Rovituso, W. Tinganelli, A. Maier, R. Kaderka, W. Kraft-Weyrather, S. Brons, T. Tessonnier, K. Parodi, M. Durante, Helium ions for radiotherapy? Physical and biological verifications of a novel treatment modality: Helium ions for radiotherapy?, Med. Phys. 43 (2016) 1995-2004. https://doi.org/10.1118/1.4944593.

[5] B. Knäusl, H. Fuchs, K. Dieckmann, D. Georg, Can particle beam therapy be improved using helium ions? a planning study focusing on pediatric patients, Acta Oncologica. $55 \quad$ (2016) 751-759. https://doi.org/10.3109/0284186X.2015.1125016.

[6] W.D. Newhauser, R. Zhang, The physics of proton therapy, Phys. Med. Biol. 60 (2015) R155-R209. https://doi.org/10.1088/0031-9155/60/8/R155.

[7] E. Fokas, G. Kraft, H. An, R. Engenhart-Cabillic, Ion beam radiobiology and cancer: Time to update ourselves, Biochimica et Biophysica Acta (BBA) Reviews on Cancer. 1796 (2009) 216-229. https://doi.org/10.1016/j.bbcan.2009.07.005.

[8] V. Anferov, I.J. Das, Biological Dose Estimation Model for Proton Beam Therapy, IJMPCERO. 04 (2015) 149161. https://doi.org/10.4236/ijmpcero.2015.42019. 
[9] M. Cunha, C. Monini, E. Testa, M. Beuve, NanOx, a new model to predict cell survival in the context of particle therapy, Phys. Med. Biol. 62 (2017) 1248-1268. https://doi.org/10.1088/1361-6560/aa54c9.

[10] M. Beuve, A. Colliaux, D. Dabli, D. Dauvergne, B. Gervais, G. Montarou, E. Testa, Statistical effects of dose deposition in track-structure modelling of radiobiology efficiency, Nuclear Instruments and Methods in Physics Research Section B: Beam Interactions with Materials and Atoms. 267 (2009) 983988. https://doi.org/10.1016/j.nimb.2009.02.016.

[11] G. Giovannini, T. Böhlen, G. Cabal, J. Bauer, T. Tessonnier, K. Frey, J. Debus, A. Mairani, K. Parodi, Variable RBE in proton therapy: comparison of different model predictions and their influence on clinical-like scenarios, Radiat Oncol. 11 (2016) 68. https://doi.org/10.1186/s13014-016-0642-6.

[12] C. Koumeir, V. De Nadal, R. Cherubini, M. Cherel, E. Garrido, S. Gouard, A. Guertin, F. Haddad, V. Metivier, N. Michel, F. Poirier, N. Servagent, T. Soulanet, N. Varmenot, The radiobiological platerform at at ARRONAX, Radiat Prot Dosimetry. 183 (2019) 270273. https://doi.org/10.1093/rpd/ncy301.

[13] F. Haddad, L. Ferrer, A. Guertin, T. Carlier, N. Michel, J. Barbet, J.-F. Chatal, ARRONAX, a high-energy and high-intensity cyclotron for nuclear medicine, Eur J Nucl Med Mol Imaging. 35 (2008) 1377-1387. https://doi.org/10.1007/s00259-008-0802-5.

[14] M. Ghergherehchi, H. Afarideh, M. Ghannadi, A. Mohammadzadeh, G.R. Aslani, B. Boghrati, Proton Beam Dosimetry: a Comparison between a Plastic Scintillator, Ionization Chamber and Faraday Cup, JRR. 51 (2010) 423-430. https://doi.org/10.1269/jrr.09121.

[15] A. Subercaze, A. Guertin, F. Haddad, L. Jean-Soro, C. Koumeir, V. Metivier, N. Michel, C. Neel, A. Rahmani, N. Servagent, Une plateforme pour l'analyse de matériaux par faisceaux d'ions à ARRONAX. Étude de l'effet d'humidité sur les échantillons, isi. 15 (2016) 117-127. https://doi.org/10.3166/i2m.15.3-4.117-127.

[16] J. Krimmer, D. Dauvergne, J.M. Létang, É. Testa, Prompt-gamma monitoring in hadrontherapy: A review, Nuclear Instruments and Methods in Physics Research Section A: Accelerators, Spectrometers, Detectors and Associated Equipment. $878 \quad$ (2018) 58-73. https://doi.org/10.1016/j.nima.2017.07.063.

[17] J. Smeets, F. Roellinghoff, D. Prieels, F. Stichelbaut, A. Benilov, P. Busca, C. Fiorini, R. Peloso, M. Basilavecchia, T. Frizzi, J.C. Dehaes, A. Dubus, Prompt gamma imaging with a slit camera for real-time range control in proton therapy, Phys. Med. Biol. 57 (2012) 3371-3405. 9155/57/11/3371.

[18] J.M. Verburg, J. Seco, Proton range verification through prompt gamma-ray spectroscopy, Phys. Med. Biol. 59 (2014) 7089-7106. https://doi.org/10.1088/00319155/59/23/7089.

[19] K. Parodi, On- and off-line monitoring of ion beam treatment, Nuclear Instruments and Methods in Physics Research Section A: Accelerators, Spectrometers, Detectors and Associated Equipment. 809 (2016) 113119. https://doi.org/10.1016/j.nima.2015.06.056.

[20] M. Yamaguchi, K. Torikai, N. Kawachi, H. Shimada, T. Satoh, Y. Nagao, S. Fujimaki, M. Kokubun, S. Watanabe, T. Takahashi, K. Arakawa, T. Kamiya, T. Nakano, Beam range estimation by measuring bremsstrahlung, Phys. Med. Biol. 57 (2012) 2843-2856. https://doi.org/10.1088/0031-9155/57/10/2843.
[21] M. Yamaguchi, K. Torikai, N. Kawachi, H. Shimada, T. Satoh, Y. Nagao, S. Fujimaki, M. Kokubun, S. Watanabe, T. Takahashi, K. Arakawa, T. Kamiya, T. Nakano, Corrigendum: Beam range estimation by measuring bremsstrahlung (2012 Phys. Med. Biol . 57 2843), Phys. Med. Biol. 61 (2016) 3638-3644. https://doi.org/10.1088/0031-9155/61/9/3638.

[22] M. Yamaguchi, Y. Nagao, K. Ando, S. Yamamoto, T. Toshito, J. Kataoka, N. Kawachi, Secondary-electronbremsstrahlung imaging for proton therapy, Nuclear Instruments and Methods in Physics Research Section A: Accelerators, Spectrometers, Detectors and Associated Equipment. 833 (2016) 199-207. https://doi.org/10.1016/j.nima.2016.07.034.

[23] M. Yamaguchi, Y. Nagao, N. Kawachi, T. Satoh, S. Fujimaki, T. Kamiya, K. Torikai, H. Shimada, H. Sugai, M. Sakai, K. Arakawa, T. Nakano, Detection of a gas region in a human body across a therapeutic carbon beam by measuring low-energy photons, Int. J. PIXE. 26 (2016) 61-72. https://doi.org/10.1142/S0129083516500078.

[24] L. Schwob, C. Koumeir, N. Servagent, M. Cherel, A. Guertin, F. Haddad, V. Métivier, N. Michel, F. Poirier, A. Rahmani, N. Varmenot, New beam monitoring tool for radiobiology experiments at the cyclotron ARRONAX, Radiat Prot Dosimetry. 166 (2015) 257 260. https://doi.org/10.1093/rpd/ncv199.

[25] K. Ishii, Continuous X-rays produced in light-ion-atom collisions, Radiation Physics and Chemistry. 75 (2006) $1135-1163$. https://doi.org/10.1016/j.radphyschem.2006.04.008.

[26] J.E. Miraglia, Scaling laws for secondary-electron bremsstrahlung, Phys. Rev. A. 39 (1989) 2908-2913. https://doi.org/10.1103/PhysRevA.39.2908.

[27] M.C. Pacher, J.E. Miraglia, Scaling laws and polarization of atomic and nuclear bremsstrahlung, Phys. Rev. A. 41 (1990) 2574-2579. https://doi.org/10.1103/PhysRevA.41.2574.

[28] T.C. Chu, K. Ishii, A. Yamadera, M. Sebata, S. Morita, Quasifree electron bremsstrahlung induced by $20-\mathrm{MeV}$ proton impact, Phys. Rev. A. 24 (1981) 1720-1725. https://doi.org/10.1103/PhysRevA.24.1720.

[29] K. Ishii, K. Hitomi, Theoretical detection limit of PIXE analysis using $20 \mathrm{MeV}$ proton beams, Nuclear Instruments and Methods in Physics Research Section B: Beam Interactions with Materials and Atoms. 417 (2018) $37-40$. https://doi.org/10.1016/j.nimb.2017.10.008.

[30] J.H. Hubbell, S.M. Seltzer, X-Ray Mass Attenuation Coefficients, NIST. (2009) https://www.nist.gov/pml/x-ray-mass-attenuationcoefficients (accessed May 8, 2021).

[31] B.P. Mohanty, P. Balouria, M.L. Garg, T.K. Nandi, V.K. Mittal, I.M. Govil, Comparison of experimental and theoretical efficiency of HPGe X-ray detector, Nuclear Instruments and Methods in Physics Research Section A: Accelerators, Spectrometers, Detectors and Associated Equipment. 584 (2008) 186-190. https://doi.org/10.1016/j.nima.2007.10.017.

[32] K. Moribayashi, Simulation study of radial dose due to the irradiation of a swift heavy ion aiming to advance the treatment planning system for heavy particle cancer therapy: The effect of emission angles of secondary electrons, Nuclear Instruments and Methods in Physics Research Section B: Beam Interactions with Materials $\begin{array}{llll}\text { and Atoms. } & 365 \quad \text { (2015) 592-595 }\end{array}$ https://doi.org/10.1016/j.nimb.2015.10.028. 
[33] M. Hollmark, J. Uhrdin, D. Belki, I. Gudowska, A. Brahme, Influence of multiple scattering and energy loss straggling on the absorbed dose distributions of therapeutic light ion beams: I. Analytical pencil beam model, Phys. Med. Biol. 49 (2004) 3247-3265. https://doi.org/10.1088/0031-9155/49/14/016.

[34] M.J. Berger, J.S. Coursey, M.A. Zucker, J. Chang, S.M. Seltzer, P.M. Bergstrom, Stopping-Power \& Range Tables for Electrons, Protons, and Helium Ions, NIST. (2009). https://www.nist.gov/pml/stopping-powerrange-tables-electrons-protons-and-helium-ions (accessed May 8, 2021). 